\title{
EN TORNO AL 'FILOHELENISMO' DE LOS ESCOLIOS HOMÉRICOS
}

\author{
Jaume Pòrtulas \\ Universitat de Barcelona \\ jportulas@ub.edu
}

\section{RESUMEN}

Los comentadores antiguos de la Ilíada substituían la dicotomía homérica "aqueos vs. troyanos" por "griegos vs. bárbaros". Los estudiosos modernos ya no denominan 'bárbaros' a los troyanos; pero suelen contraponer troyanos y 'griegos', obviando las denominaciones originales de aqueos, dánaos, etc. Esta distorsión no es inocua: da por supuesto, sin discusión, que los auditorios de la Ilíada experimentaban una afinidad especial por un bando y lo identificaban con "los nuestros". No es imposible que este sentimiento de afinidad existiera a veces; pero resulta abusivo interpretarlo en sentido identitario, étnico o 'patriótico'. Sin embargo, esto es lo que suelen hacer los escolios, con grave distorsión, en bastantes casos, del texto original.

Palabras Clave: Escolios, Ilíada, filohelenismo, etnicidad, bárbaros.

\section{SOME THOUGHTS ON 'PHILHELLENISM’ AND HOMERIC SCHOLIA}

\section{ABSTRACT}

Iliad's ancient commentators replaced the Homeric dichotomy "Achaeans vs. Trojans" with "Greeks vs. Barbarians”. Modern scholars no longer call the Trojans 'barbarians'; but they usually oppose Trojans and 'Greeks', dispensing with the original denominations of Achaeans, Danaans, etc. This distortion is not innocuous: it assumes without discussion that the Iliad's ancient audiences experienced a special affinity for one side and identified it with "our side". It is not impossible that this feeling existed at times; but it is abusive to interpret it in an ethnic, national or 'patriotic' sense. However, this is what the Scholia usually do, seriously distorting, in many cases, the original text.

KEYWORDS: Scholia, Iliad, philhellenism, ethnicity, barbaroi.

1. Voy a tomar como punto de partida para estos apuntes -redactados a vuelapluma, para el homenaje a un querido amigo- un hecho bien conocido, y generalmente aceptado como obvio, hasta el punto de que no se le suele prestar ninguna atención. Me refiero a que los comentadores antiguos de la Ilíada-desde los escoliastas que resumen la doctrina de los filólogos helenísticos hasta Eustacio de Tesalónica- traducen unánimemente la dicotomía «aqueos (+ dánaos, argivos, etc.) vs. troyanos» por 
"griegos vs. bárbaros". Ésta es también la práctica normal de los autores griegos (clásicos, helenísticos y de Época imperial), cuando se refieren al famoso conflicto y a su cantor. Pero el término $\beta \alpha \alpha \rho \beta \alpha \rho o \varsigma$, como todo el mundo sabe, no es en absoluto homérico. En 'Homero', sólo aparece el adjetivo $\beta \alpha \rho \beta \alpha \rho$ ó $\varphi \omega v o \varsigma$, y además una sola vez, aplicado a los carios de Mileto, con el sentido etimológico de "hablantes de una lengua distinta, incomprensible". Por lo demás, en el imaginario de la Ilíada, aqueos y troyanos hablan, evidentemente, la misma lengua ${ }^{2}$.

Los estudiosos modernos, obviamente, ya no se refieren a los troyanos como "los bárbaros"; sin embargo, siguen utilizando el término 'griegos' (quizás por comodidad) como primer elemento de la dicotomía "griegos vs. troyanos», en vez de las correspondientes voces homéricas: aqueos, dánaos, argivos, etcétera. Ahora bien, el término 'griegos' no es tampoco homérico, propiamente. Las designaciones "E $\lambda \lambda \eta \nu \varepsilon \varsigma$

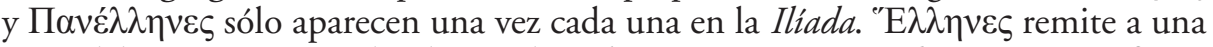
parte del contingente tesalio de Aquiles (Il. II 684). Los $\Sigma$ D (ad IX 395) especifican: "Según Aristarco, quien sigue en esto a Tucídides [i 3, 1-3], 'la Hélade', en Homero,

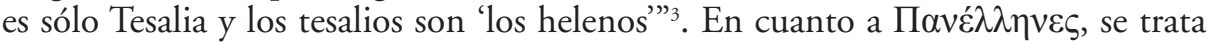
de un uso absolutamente aislado (Il. II 530); e incompatible, además, con las otras apariciones de ${ }^{5} \lambda \lambda \alpha^{\alpha} \varsigma$ en los poemas (que también son escasas) ${ }^{4}$. Así pues, Aristarco condenó los dos $v v$. 529-530 como espúreos. Los editores modernos, tanto si los aceptan como si los rechazan, acostumbran a subrayar que el sentido ampliado de ПаvÉ $\lambda \lambda \eta v \varepsilon \varsigma$ refleja un desarrollo tardío5.

Ahora bien, recurrir a una designación ajena al texto original (o, para ser más exactos, apenas existente en dicho texto, y con un significado distinto) es una opción poco aconsejable a priori, y que conlleva riesgos considerables, desde la arbitrariedad hasta la tergiversación. Pero mi intención no es abogar ahora por la supresión de una práctica tan arraigada, aunque ciertamente incorrecta; sólo me propongo llamar la atención sobre los problemas que el uso semi-automático del término 'griegos' para referirse a uno de los grupos enfrentados en la Ilíada suscita en la exégesis homérica. Me refiero, en concreto, a inconvenientes de dos tipos:

1. Se presupone un vínculo emocional intenso -y de tipo, digamos, 'patriótico'entre determinados personajes de la epopeya (abusivamente denominados 'griegos')

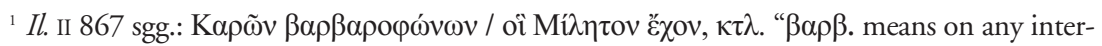
pretation of $\beta \alpha \dot{\alpha} \rho \beta \alpha \rho \varsigma \varsigma$ that they do not speak Greek — which is surprising since Miletos was a Minoan foundation certainly inhabited by Mycenaean Greeks towards the end of the Bronze Age” (Kirk, 1985: 260).

${ }^{2}$ Cosa que no ha sido óbice para la producción de interesantes propuestas de diferenciar el uso que hacen unos y otros de esta misma lengua. Véase e.g. Mackie, 1996.

${ }^{3}$ Véase Schironi, 2018: 301.

${ }^{4}$ Cinco veces en la $I l$. (siempre refiriéndose a la región de Ftia) y cuatro en $\mathrm{Od}$. (donde parece remitir a la Grecia Central y del Norte, en contraposición al Peloponeso). Cf. Kirk, 1985: 202.

${ }^{5}$ Vid. e.g. Kirk, 1985: 202: "The meaning has been extended still further to cover the Greeks

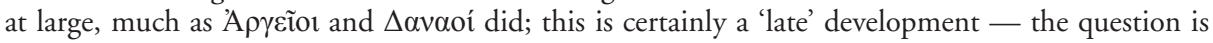
whether it is a post-Homeric one". 
y los auditorios antiguos. A priori, no es imposible que este vínculo existiera, por lo menos en algunos casos. Pero resulta poco metódico darlo por supuesto de entrada, sin necesidad siquiera de plantearse la cuestión claramente. Por otra parte, hay mucho que decir acerca del carácter presuntamente 'patriótico' del vínculo en cuestión. 2. Identificar a los 'aqueos' con 'los griegos' contribuye a enmarañar las discusiones sobre la vexata quaestio del filohelenismo (otros dicen directamente 'el patriotismo') del poeta (o los poetas) de la Ilíada, como se verá a continuación.

Con la identificación automática, y sin análisis previo, «aqueos = griegos», las cosas tienden a quedar muy claras; demasiado claras, en realidad. Los aqueos serían los protagonistas del epos; es decir, "los nuestros". No hace falta subrayar las múltiples distorsiones que una identificación de este tipo provoca de modo subrepticio en la exegesis de una serie de pasajes; y de modo especial (aunque no único) en la interpretación de los episodios bélicos.

2. Los escolios homéricos constituyen un ámbito particularmente adecuado para observar semejantes distorsiones ${ }^{6}$. Me refiero a los $\Sigma \mathrm{bT}$, pues A y D inciden muy poco en esta problemática. En bT, en cambio, hallamos repetidas veces el tajante postulado

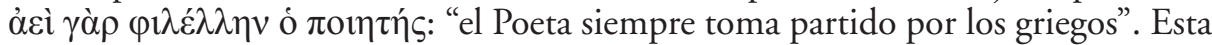
expresión se repite en diversas ocasiones; por ejemplo, a propósito de X 14-16 y de XVI 814, y también en la introducción al libro XI.

Recordaré rápidamente estos pasajes. En $\mathrm{Il}$. x 14-16 se describe la desesperación de Agamenón al contemplar los efectos de la derrota aquea y el triunfo de los troyanos. Pero, según los escolios, el poeta no se demora detallando los temores del rey (afirmación francamente inexacta); por amor de patria, se limita a contrastar la alegría de los unos y el desánimo de los otros. En cuanto a XVI 814-15, se refiere a Euforbo, el troyano que se da a la fuga después de herir a Patroclo por la espalda -con la imprescindible colaboración de Apolo. El escoliasta parece opinar que la preocupación fundamental del poeta es "ridiculizar $(\kappa \alpha \tau \alpha \kappa \omega \mu \omega \delta \tilde{\omega} \nu)$ al bárbaro". ¿Y por qué no a Apolo, si nos situamos en esta tesitura? Parece difícil ofrecer resúmenes más sesgados de ambos episodios. Por lo que respecta al extenso libro XI, éste se abre, como se recordará, con victorias importantes de Agamenón y otros paladines aqueos; mas uno tras otro van cayendo heridos, y el canto se cierra en pleno desastre para su bando. Los escolios opinan que semejante presentación es producto del 'filohelenismo' de Homero, un sentimiento que no le permitiría narrar los contratiempos de 'los suyos' sin haberlos presentado antes bajo una luz rotundamente favorable.

Me parece que no hace falta insistir demasiado en el hecho de que el axioma

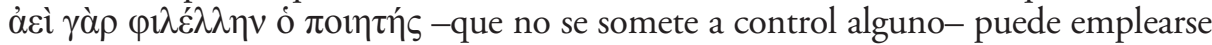
para forzar el texto de la Ilíada de un modo más bien brutal. Sin embargo, me gustaría presentar un par de ejemplos complementarios de semejantes distorsiones, para documentar mejor su carácter.

${ }^{6}$ Cf. Kakridis, 1971: 54-67. 
A propósito del choque entre Agamenón y Crises, en el canto I, el escoliasta a los vv. 29-31 anota que, si hubiera entendido bien la cosa, Crises no se habría ofendido, sino más bien alegrado, al enterarse de que su hija pasaría a ser propiedad del gran rey ${ }^{7}$. En tal observación (desacomplejadamente sexista, por otra parte), se conjugan un sentimiento enérgico de la superioridad griega (ajeno al texto, por lo menos a mi entender) y una devoción por el poder regio que todavía le es más ajena, si cabe. Oliver Taplin (1990: 81) comenta, no sin cierta acidez, que el punto de vista del escoliasta "reflects the mores of Hellenistic monarchs rather than of Homer". Se podrá discutir si esta drástica reinterpretación de la escena corresponde a los eruditos de Alejandría o es más bien obra de los escoliastas bizantinos; pero esta cuestión no es relevante para nuestro argumento de hoy.

Veamos otro ejemplo, que afecta al mundo de los dioses. En XV 69-77, Zeus cuenta detalladamente sus planes para el desarrollo futuro de la acción a su esposa, la diosa Hera, quien, hasta el momento presente, se había mostrado particularmente insumisa. No resulta fácil decidir si Zeus le da tales explicaciones para tranquilizarla, o para obtener su complicidad, o más bien para acabar de humillarla con todo el peso de su superior previsión. En cualquier caso, el escolio opina que, en realidad, a quien el padre de los dioses pretende tranquilizar no es tanto a su esposa como, directamente, a los oyentes del poema ${ }^{8}$ :

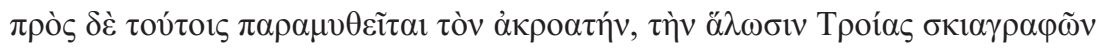

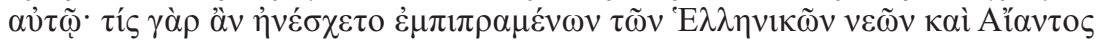

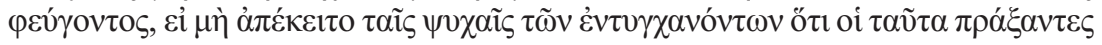

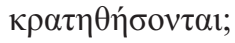

... además, [el Poeta] tranquiliza a su oyente con un apunte de la toma de Troya. Pues ¿quién soportaría el incendio de las naves griegas y la huída de Ayante, si los presentes no tuvieran en sus corazones la seguridad de que los responsables de tales acciones serían al fin derrotados?

Pero, en realidad, no tenemos el menor indicio objetivo en el sentido de que los antiguos auditorios de la Ilíada encajaran con tanta incomodidad como el escoliasta $-\mathrm{O}$, por decir mejor, sus fuentes- las numerosas derrotas y humillaciones que Héctor y los troyanos infligen a los aqueos en ausencia de Aquiles. Ésta es una reacción que se acepta "como natural" de modo apriorístico.

3. En realidad, el hecho de que los primeros receptores de los poemas homéricos, esto es, los ciudadanos de las poleis arcaicas, se consideraban en cierto sentido descendientes y herederos de los debeladores de Troya no resulta dudoso. Pero también está muy

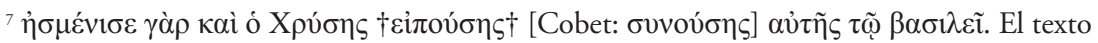
está corrupto, pero el sentido resulta perfectamente claro.

${ }^{8} \Sigma \mathrm{bT}$ ad 56b, 16-19 (IV 22 Erbse). 
claro que su sentido de pertenecer a una comunidad, su 'patriotismo' -utilizo ahora, de modo deliberado, un término anacrónico- se vinculaba sobre todo a la polis de cada uno; sólo de un modo tendencial e incipiente asumía una dimensión panhelénica. Las performances homéricas (de las que nos podemos formar cierta idea a partir de las Panateneas atenienses, a pesar de su origen no excesivamente antiguo) contribuyeron de modo importante, y quizás decisivo, al surgimiento de dicha conciencia? Pero es difícil fechar este desarrollo antes de la segunda mitad del siglo VI a.C., cuando se empieza a dibujar la amenaza 'oriental' sobre la Grecia peninsular. Ahora no podemos entrar, por razones obvias, en la compleja cuestión de la cronología de los poemas; pero nos basta con afirmar que no hay que considerarlos testimonio de la conciencia panhelénica sino como uno de los factores que contribuyeron a crearla ${ }^{10}$. Actualmente, se suele admitir que la contraposición entre griegos y bárbaros corresponde a la Época clásica, no al Arcaísmo: cristalizó al calor de las Guerras Médicas y de la victoria griega -que, por otra parte, sólo a posteriori devino propiamente 'griega' ${ }^{11}$. En la tragedia ática es donde hallamos articulado por vez primera un contraste tajante entre el bárbaro y el heleno 'superior' ${ }^{12}$.

Además, el hecho de considerarse "herederos de los héroes de Troya" no inducía en modo alguno a los ciudadanos de las diversas poleis a renunciar a sus respectivos mitos locales de origen -la autoctonía en el caso de los atenienses, el retorno de los Heráclidas en el de los espartanos, Cadmo y la siembra de los dientes del dragón en Tebas, etc. ${ }^{13}$. Y todo ello, a pesar de que la coexistencia de las distintas leyendas epicóricas y su encaje con la gesta troyana suscitaba no pocas complicaciones lógicas y cronológicas.

La conexión con Troya era reivindicada, pues, por los griegos arcaicos no tanto en calidad de participantes en una empresa colectiva, sino como ciudadanos de cada una de las ciudades-estado. Recordaré, en este sentido, una sugestiva anécdota, narrada por Heródoto (VII 158). En vísperas de la expedición de Jerjes contra Atenas y Esparta, embajadores de ambas ciudades acudieron a Gelón de Siracusa en demanda de ayuda. Pero los espartanos se negaron a ceder a Gelón el mando

\footnotetext{
${ }^{9}$ Sobre las Panateneas me limitaré a citar, entre una bibliografia muy copiosa, Davison, 1958 : 23-42; Neils, 1992; Calame, 1996: 470-80; Nagy, 2002; Pòrtulas, 2008: 474 sgg.

${ }^{10}$ Sobre la construcción de un "Homero 'panhelénico", e.g. Graziosi, 2002: 86-87, 196-99; Nagy, 2010: 9-28, 218-253.

${ }^{11}$ Cf. e.g. J. M. Hall, 2002: 172-228.

${ }^{12}$ Tesis mantenida por Edith Hall, 1989, un trabajo que ha alcanzado rápidamente estatus de canónico.

${ }^{13}$ Los mitos de origen de las diversas poleis griegas han sido objeto de muchos estudios importantes; en el contexto presente, me limitaré a una breve lista de trabajos representativos. Para Atenas: Burkert, 1966: 1-25; Loraux, 1990²; Detienne, 2003. Esparta: Calame, 1987: 153-186. Tebas: Vian, 1963. Por otra parte, Sabbatucci, 1978: 73-169 reflexiona (desde una perspectiva histórico-religiosa) acerca de la riqueza y complejidad de los mitos de origen epicóricos en contraste con la relativa pobreza de los panhelénicos.
} 
supremo de la empresa -a pesar de que su apabullante superioridad en hombres, armas, barcos y dinero le convertía en el candidato obvio-, apelando nada menos que al vínculo especial de Esparta con Agamenón, el comandante supremo de la antigua expedición aquea. En realidad, tanto la naturaleza del mando de Agamenón como sus conexiones con la futura polis de Esparta tenían escasa relación con las pretensiones del embajador espartano; pero eso es lo de menos. Lo que ahora importa es el uso partidista y sesgado que hacía Esparta de su relación con la empresa troyana.

El interés de los atenienses (cuya participación en los hechos narrados por la Ilíada resulta menos que modesta) por magnificar su papel en Troya también confirma que la conexión con el mundo de los héroes era percibida en términos de polis, no de colectividad panhelénica. No voy a evocar aquí las singulares anécdotas en torno a las interpolaciones perpetradas en el texto homérico por Pisístrato y/o sus hijos y colaboradores, con la intención de reforzar, en la medida de lo posible, los vínculos entre la saga troyana y su propia ciudad ${ }^{14}$. Ahora no importa dilucidar si tales anécdotas son o no verídicas; en la versión que se nos ha transmitido, probablemente no lo sean. Lo que interesa para nuestra argumentación actual es la construcción de una relación 'patriótica' entre la Ilíada y sus receptores (sus receptores atenienses, en este caso concreto). Acerca del carácter secundario, y hasta cierto punto tardío, de la operación, caben muy pocas dudas.

Quizás el ejemplo más ilustrativo (transmitido también por Heródoto, en V 67) es el del tirano Clístenes de Sición. En torno al 570 a.C., este personaje, exasperado por el hecho de que las performances de los rapsodas contenían (desde su punto de vista) excesivos elogios de Argos y los argivos, enemigos tenaces de los sicionios, adoptó la medida extrema de prohibir las recitaciones rapsódicas en todo el territorio de Sición. Clístenes era incapaz, al parecer, de comprender y valorar la dimensión panhelénica que la epopeya iba adquiriendo paulatinamente; en cambio, le parecía clarísimo que favorecía de un modo abusivo a sus detestados enemigos de Argos ${ }^{15}$.

4. Desde nuestra perspectiva de hoy, la conexión genealógica con personajes iliádicos más singular e interesante es la establecida con... los troyanos. Existen ciertos indicios, en efecto, en el sentido de que algunos habitantes de la Tróade (parcialmente griegos, o por lo menos helenizados), durante la época arcaica y las sucesivas, se jactaban de descender de los antiguos y prestigiosos señores del lugar. Voy a repasar sumariamente los más significativos entre estos indicios. En general, son inciertos e inconcluyentes; han suscitado numerosas polémicas. Pero no es su consistencia histórica

${ }^{14}$ Pueden encontrarse discusiones y abundante bibliografia acerca de estos problemas en Jensen, 1980: 128-71, 207-226; S.L. West, 1988: 36-39; Seaford, 1994: 144-154; Haslam, 1997: 82-84; Graziosi, 2002: 228-231; Pòrtulas, 2008: 471-481.

${ }^{15}$ Análisis diversos de este relato en Svenbro, 1984: 54; Nenci, 1994: 257; Burkert, 2001: 175-76, 201, 211; Pòrtulas, 2008: 253, 454-55. 
lo que interesa realmente, sino el simple hecho de que unas conexiones de tal naturaleza hayan sido postuladas y hayan merecido una consideración seria.

A partir de ciertas informaciones del libro XIII de la Geografia de Estrabón de Amasia (circa 64-63 a.C. - 19/24 d.C.) se ha deducido que vivía en la Tróada, en época "de "Homero", una familia reinante de presuntos descendientes de Eneas ${ }^{16}$. Existe también un pasaje famoso del canto XX de la Ilíada (vv. 306-308), donde el dios Poseidón, a pesar de su habitual militancia pro-aquea, declara que es preciso salvar la vida de Eneas, quien está corriendo un riesgo inútil y descabellado intentando enfrentarse con Aquiles. Poseidón también afirma que es voluntad de Zeus que, una vez saqueada Troya y exterminados Príamo y sus descendientes directos, Eneas y los suyos continúen señoreando aquellos lugares. Estos versos han sido considerados como una noticia, traspuesta en profecía, sobre un oĩko dientes de Eneas que habrían sobrevivido en la Tróada en tiempos del poeta: un oĩ absolutamente desconocido, por otra parte, si descontamos la fácil (y atractiva) conexión con la noticia de Estrabón a la que nos acabamos de referir ${ }^{17}$. Bastantes estudiosos modernos se han arriesgado a conjeturar, incluso, que uno de estos descendientes de Eneas pudo haber sido el patrocinador, el mecenas, de cierto poeta llamado Homero... Robert Wood, en su An Essay on the Original Genius of Homer (Londres 1769), ya conjeturaba que la profecía de Poseidón remitía a esta noble familia; y Karl Otfried Müller (1797-1840), entre otros, articuló la hipótesis del mecenazgo. Ambas propuestas han gozado -habitualmente en combinación- de un predicamento considerable en los estudios históricos y filológicos de los siglos XIX y $\mathrm{XX}^{18}$.

5. Las hipótesis de este tipo puede ser más o menos atractivas, incluso fascinantes; en cualquier caso, resultan indemostrables, por lo menos en el estado actual de nuestros conocimientos $^{19}$. Ahora bien todas estas construcciones demuestran de sobras - por lo menos en mi opinión- que, como apuntábamos al principio, la dicotomía "aqueos

${ }^{16}$ Vid. Estrabón XIII \$1 52-53 (III 607 fCasaubon). La fuente del geógrafo es Demetrio de Escepsis (una ciudad de la misma Tróada). Puede hallarse un análisis minucioso de toda la cuestión en Smith, 1981: 34-43, quien, por otra parte, considera tales noticias del todo indignas de crédito. Según este estudioso, la leyenda habría surgido de una sobreinterpretación de los versos del Ilíada XX que comentamos acto seguido, en combinación con determinados pasajes del Himno homérico a Afrodita (vid. n. siguiente).

${ }^{17}$ También hay que tener en cuenta, claro está, los pasajes paralelos del Himno homérico a Afrodita (vid. n. anterior); pero resulta fácil descartarlos, atribuyéndolos a la influencia del epos mayor.

${ }^{18}$ Bosquejos históricos muy interesantes del desarrollo de toda la cuestión - de la que Reinhardt, 1956 (cf. Reinhardt, 1961: 450-53; 507-521) constituye, quizás, el momento culminante- pueden hallarse e.g. en Smith, 1981: 17-25 y en Faulkner, 2008: 3-10.

${ }^{19}$ Entre sus defensores recientes se cuentan Edwards, 1991: 299-301; Faulkner (cit. en la n. anterior), ambos con muchos matices; y M. L. West, 2011: 26. Se muestran escépticos, en cambio, Smith, 1981 y J. S. Clay, 1989: 153, entre otros. 
vs. troyanos" no es recogida de modo adecuado por la contraposición "griegos vs. bárbaros"; ni tampoco por su sucesora, "griegos vs. troyanos". Para que semejantes dicotomías tengan sentido, resulta imprescindible, claro está, que las nociones de 'griego' y 'bárbaro' hayan cristalizado; y ya vimos que no era éste el caso en la Grecia 'homérica' (ni tampoco en el primer Arcaísmo). No cabe duda, por otra parte, de que, cuando ambas nociones se consolidaron, fue de modo simultáneo, como cara y cruz de una misma moneda.

En el seno de nuestra Ilíada, tanto los aqueos -llamados también dánaos, argivos, etc.- como los troyanos son colectividades míticas. Al utilizar este término, polisémico y comprometido, me refiero sobre todo al hecho de que se trata de personajes separados del poeta (o poetas) y de los auditorios iniciales por un hiato que no es únicamente temporal. Como sucede con todos los héroes, resulta perfectamente lícito, en determinadas circunstancias, vincularse a ellos por la vía (pseudo-)genealógica ${ }^{20}$. Al fin y al cabo, constituyen una suerte de antepasados colectivos nocionales, como se dice en inglés: nocionales, no nacionales. Y, en tanto que antepasados míticos, aqueos y troyanos difieren más bien poco. Prueba de ello es que las colectividades posteriores (griegas o helenizadas) podían contemplar la vinculación genealógica con unos y con otros, de acuerdo con razones circunstanciales. Pero es que tampoco se relacionaban con estos supuestos antepasados en tanto que 'helenos', sino como espartanos, atenienses, argivos o habitantes de la Tróade: como miembros, pues, de determinadas colectividades que tributaban honores a sus héroes/antepasados.

6. Para terminar, me gustaría volver un instante a mi punto de partida: la impropiedad de substituir masivamente, en los estudios y comentarios homéricos, los términos 'aqueos', 'dánaos', etc. por 'griegos', y los malentendidos que semejante impropiedad suscita. No voy a defender, desde luego, que los poeta(s) y auditorios homéricos no percibieran ningún tipo de diferencia entre los bandos enfrentados en la guerra de Troya. Pero no se trata de diferencias socio-antropológicas (como podría sugerir la dicotomía "griegos vs. bárbaros"), ni tampoco 'nacionales' y/o culturales (implícitas en una contraposición como "griegos vs. troyanos"). Para recurrir, como me parece oportuno en este caso, a un ejemplo puramente mítico, aunque sea lejano, quizás se podría aducir el enfrentamiento entre los Pāṇdava y los Kaurava en el Mahābhārata. Los enemigos encarnizados de la gran epopeya sánscrita resultan muy próximos entre sí: son primos hermanos -nacidos, además, en el seno de una dinastía profundamente endogámica, que coquetea muchas veces con el incesto. Al mismo tiempo,

${ }^{20}$ Sobre la compleja relación entre héroes y antepasados, cf. e.g. Brelich, 1958: 141-51, 196 sgg., 269-70, etc.; Nagy, 2013: 256 sgg., 486 sgg. Véase también J. M. Hall, 1997, una fascinante demostración de hasta qué punto las designaciones grupales griegas como 'dorios', 'jonios' o 'argivos' no responden a auténticas realidades étnicas, lingüísticas o culturales sino a construcciones ideológicas de la época clásica, al servicio de distintos proyectos políticos. 
los hijos de Pandu son avatara (en el sentido propio de "encarnaciones mortales") de los deva (los dioses), mientras que los Kaurava lo son de los enemigos de los dioses, los asura ${ }^{21}$.

De un modo hasta cierto punto comparable, las oposiciones básicas de la Ilíada no parecen responder, en sus estratos profundos, a la lógica de un choque entre $\varepsilon \ddot{\theta v \varepsilon \alpha}$ distintos, o entre naciones rivales; ni tampoco entre Occidente y Oriente; o entre pueblos civilizados y pueblos bárbaros. Podríamos decir que Troya es una ciudad esencialmente, ontológicamente condenada; pero las culpas 'objetivas' de sus habitantes (culpas perfectamente 'reales', por otra parte; piénsese en el rapto de Helena y los episodios concomitantes) asumen muchas veces un carácter sorprendentemente trivial y anecdótico. Por otro lado, Aquiles es una fuerza triunfante, y a la vez destructora -destructora también, y quizás sobre todo, de los suyos- por razones que nada tienen que ver con la psicología, la moral o la nacionalidad ${ }^{22}$. No es justo reducirlo todo, como hacen los escolios bizantinos, a un enfrentamiento bélico entre "los nuestros" y "los otros".

\section{REFERENCIAS BIBLIOGRÁFICAS}

BIARDEAU, M. (2002): Le Mahābhārata. Un récit fondateur de brahmanisme et son interprétation (2 vols.), Éditions du Seuil, Paris.

BReLICH, A. (1958): Gli eroi greci. Un problema storico-religioso, Ateneo, Roma.

BURKERT, W. (1966): «Kekropidensage und Arrhephoria. Vom Initiationsritus zum Panathenäenfest», Hermes XCIV: 1-25.

Burkert, W. (2001): Kleine Schriften I. Homerica, ed. Ch. RiedWeg, Vandenhoeck und Rupprecht, Göttingen.

CALAme, C. (1987): «Spartan Genealogies: The Mythological Representation of a Spatial Organisation», en J. Bremmer (ed.), Interpretations of Greek Mythology, Croom Helm, London \& Sidney, pp. 153-186.

CAlAme, C. (1996): «Feste, riti e forme poetiche», en S. SetTiS (ed.), I Greci. 2. Una Storia greca. I. Formazione (fino al VI secolo a. C.), Einaudi, Torino, pp. 470-496.

Clay, J. S. (1989): The Politics of Olympus. Form and Meaning in the Major Homeric Hymns, Princeton University Press, Princeton NJ.

Davison, J. A. (1958): «Notes on the Panathenaea», JHS LXXVIII: 23-42.

${ }^{21}$ Biardeau, 2002: I 223-283 analiza detalladamente las uniones anómicas y nacimientos irregulares del libro I del Mahābhārata como signos de la crisis del poder real. Para un análisis del conflicto de Kurukshetra no como una guerra humana convencional sino como un enfrentamiento cósmico entre poderes divinos, véase Dumézil, 1995: 59-285.

${ }^{22}$ Para el sentido del nombre Akhil(l)eus $\approx$ "he who has ä $\chi 0 \varsigma$ ["grief, sorrow"] for the people

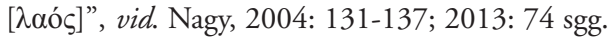


Detienne, M. (2003): Comment être autochtone, Seuil, Paris.

DiCKeY, E. (2007): Ancient Greek Scholarship, American Philological Association / Oxford University Press.

DuMÉzIL G. (1995): Mythe et Épopée I. II. III, Gallimard, Paris.

Edwards, M. W. (1991): The Iliad: A Commentary. Volume v: Books 17-20, Cambridge University Press.

FaulKner, A. (2008): The Homeric Hymn to Aphrodite. Introduction, Text, and Commentary, Oxford University Press.

Graziosi, B. (2002): Inventing Homer. The Early Reception of Epic, Cambridge University Press.

HaLl, E. (1989): Inventing the Barbarian. Greek Self-Definition through Tragedy, Clarendon Press, Oxford.

Hall, J. M. (1997): Ethnic Identity in Greek Antiquity, Cambridge University Press.

Hall, J. M. (2002). Hellenicity. Between Ethnicity and Culture, The University of Chicago Press, Chicago - London.

Haslam, M. (1997): «Homeric Papyri and the Transmission of the Text», en I. Morris \& B. Powell (eds.), A New Companion to Homer, Brill, Leiden - New York, pp. 55-100.

ERBSE, H. (1969-1988): Scholia graeca in Homeri Iliadem. Scholia vetera. (7 vols.), Walter de Gruyter, Berlin.

Jensen, M. S. (1980): The Homeric Question and the Oral-Formulaic Theory, Museum Tusculanum Press, Copenhagen.

KAKRIDIS, J. T. (1971): Homer Revisited, Gleerup, Lund.

KIRK, G. S. (1985): The Iliad: A Commentary. Volume I: Books 1-4, Cambridge University Press.

Loraux, N. (1990²): Les enfants d'Athéna. Idées athéniennes sur la citoyenneté et la division des sexes, La Découverte/Seuil, Paris.

Mackie, H. (1996): Talking Trojan. Speech and Community in the Iliad, Rowman \& Littlefield, Lanham - Boulder - New York - London.

Nagy, G. (2002): Plato's Rhapsody and Homer's Music. The Poetics of the Panathenaic Festival in Classical Athens, Center for Hellenic Studies / Harvard University Press, Washington - Athens.

Nagy, G. (2004): Homer's Text and Language, University of Illinois Press, Urbana - Chicago.

NAGY, G. (2010): Homer the Preclassic, University of California Press, Berkeley - Los Angeles - London.

NAGY, G. (2013): The Ancient Greek Hero in 24 Hours, The Belknap Press - Harvard University Press, Cambridge (MA) - London.

NeIls, J. (1992): Goddess and Polis. The Panathenaic Festival in Ancient Athens, Princeton University Press, Princeton NJ.

Nenci, G. (1994): ERODOTO. Le Storie. Libro V. La rivolta della Ionia. Fondazione Lorenzo Valla, Arnoldo Mondadori, Verona.

Pòrtulas, J. (2008): Introducció a la Ilíada. Homer entre la història i la llegenda, Fundació Bernat Metge, Barcelona.

Pinsent, J. (1984): «The Trojans in the Iliad», en L. Foxhall - J. K. Davies (eds.), The Trojan War. Its Historicity and Context, Bristol Classical Press, Bristol, pp. 141-162.

REINHARDT, K. (1956): «Zum homerischen Aphroditehymnus», en Festschrift für Bruno Snell zum 60. Geburtstag, von Freunden und Schülern überreicht, Beck, München, pp. 1-14.

ReInHARdT, K. (1961): Die Ilias und ihr Dichter. Herausgegeben vON U. HÖLSCHER, Vandenhoeck und Rupprecht, Göttingen. 
RICHARDSON, N. J. (1980): «Literary Criticism in the Exegetical Scholia to the Iliad: A Sketch», CQXXX: 265-287.

SABBATUCCI, D. (1978): Il mito, il rito e la storia, Bulzoni Editore, Roma.

SEAFORD, R. (1994): Reciprocity and Ritual. Homer and Tragedy in the Developing City-State, Clarendon Press, Oxford.

SCHIRONI, F. (2018): The Best of the Grammarians. Aristarchus of Samothrace on the Iliad, University of Michigan Press, Ann Arbor.

SMITH, P. M. (1981): «Aineiadai as Patrons of Iliad XX and the Homeric Hymn to Aphrodite», HSCP LXXXV: $17-58$.

Svenbro, J. (1984): La parola e il marmo. Alle origini della poetica greca, Boringhieri, Torino.

Taplin, O. (1990): «Agamemnon's Role in the Iliad», en C. Pelling (ed.), Characterization and Individuality in Greek Literature, Clarendon Press, Oxford, pp. 60-82.

Vian, F. (1963): Les origines de Thèbes. Cadmos et les Spartes, Klincksieck, Paris.

VAN der ValK, M. (1953): «Homer's Nationalistic Attitude», AC XXII: 5-26.

VAN DER VALK, M. (1985): «Homer's Nationalism, again», Mnemosyne XXXVIII: 373-376.

West, M. L. (2011): The Making of the Iliad. Disquisition and Analitical Commentary, Oxford University Press.

West, S. R. (1988): «The Transmission of the Text», en A. Heubeck, S. R. West \& J. B. Hainsworth, A Commentary on Homer's Odyssey. Volume I. Introduction and Books I-VIII, Clarendon Press, Oxford, pp. 33-48. 
